\title{
Investigating the versatility of multifunctional silver nanoparticles: preparation and inspection of their potential as wound treatment agents
}

\author{
Geewoo Nam ${ }^{1} \cdot$ Baskaran Purushothaman $^{1} \cdot$ Sabarinathan Rangasamy $^{1}$. \\ Joon Myong Song ${ }^{1}$
}

Received: 6 April 2015/Accepted: 16 September 2015/Published online: 11 November 2015

(C) The Author(s) 2015. This article is published with open access at Springerlink.com

\begin{abstract}
Silver nanoparticles (AgNPs) are capable of inhibiting the growth of a broad spectrum of bacterial species. The minute size of the nanoparticulates enhances their biocidal activity and is thus widely utilized as antibacterial agents. The most recently researched and recognized antibacterial and wound-healing properties of published AgNPs were investigated in this article. The following parameters of the AgNPs affecting their properties and potency were explored: particle size, shape, and type of ligand or stabilizing agent. Research regarding the antibacterial activity enhancement of high-valent silver nanoparticles compared to those of the lower valent forms were summarized and analyzed. Nanocrystalline silver is capable of binding to components that may enhance their preparation and antibacterial properties. By forming complexes with ligands that exhibit desired properties, silver nanoparticles can be synthesized to exhibit those desired properties without compromising their performance. This review will provide a detailed discussion regarding the parameter-dependent bactericidal properties of silver nanoparticles and nanocomposite silver complexes as potent multifunctional wound-healing agents.
\end{abstract}

Joon Myong Song

jmsong@snu.ac.kr

1 College of Pharmacy, Seoul National University, Seoul, South Korea

\section{Graphical Abstract}

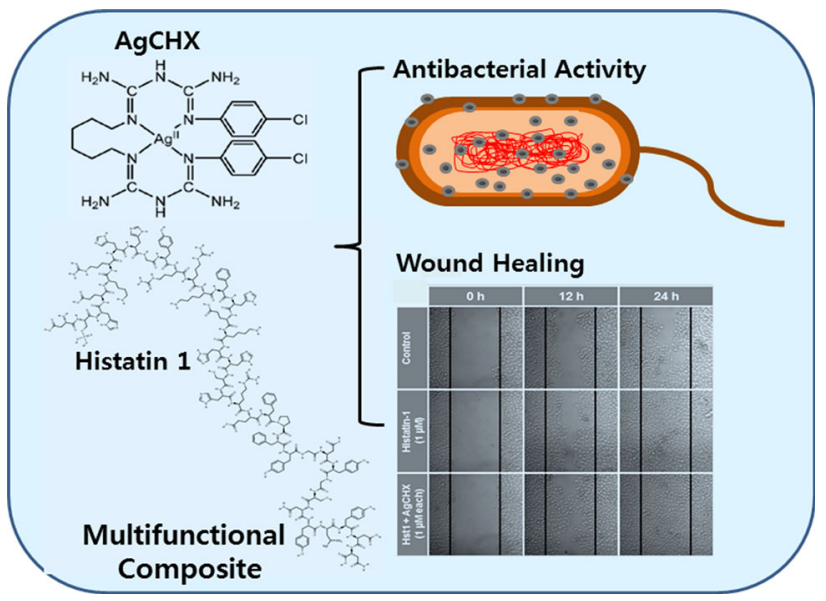

Keywords Silver nanoparticles - High-valence silver . Antibacterial · Wound healing $\cdot$ Cell proliferation

$\begin{array}{ll}\text { Abbreviations } \\ \text { AgNPs } & \text { Silver nanoparticles } \\ \text { ADP } & \text { Adenosine diphosphate } \\ \text { ATP } & \text { Adenosine triphosphate } \\ \text { SEM } & \text { Scanning electron microscopy } \\ \text { AFM } & \text { Atomic force microscopy } \\ \text { DLS } & \text { Dynamic light scattering } \\ \text { TEM } & \text { Transmission electron } \\ & \text { microscopy } \\ \text { XPS } & \text { X-ray photoelectron spectroscopy } \\ \text { XRD } & \text { Powder X-ray diffraction } \\ \text { E. coli } & \text { Escherichia coli } \\ \text { NaBH } & \text { Sodium borohydride } \\ \text { PEG } & \text { Polyethylene glycol } \\ \text { PVP } & \text { Polyvinylpyrrolidone }\end{array}$




$\begin{array}{ll}\text { MIC } & \begin{array}{l}\text { Minimum inhibitory } \\ \text { concentration }\end{array} \\ \mathrm{AgNO}_{3} & \text { Silver nitrate } \\ \mathrm{Hst}-1 & \text { Histatin-1 } \\ \mathrm{CHX} & \text { Chlorhexidine } \\ \mathrm{Ag}(\mathrm{II}) \mathrm{CHX} & \text { Silver(II) chlorhexidine complex } \\ \mathrm{ECM} & \text { Extracellular matrix }\end{array}$

\section{Introduction}

The use of silver as a medicinal substance dates back to the times of Hippocrates, the father of modern medicine who used silver's medical properties to treat wounds [1]. Historically, silver was considered one of the most important antibacterial agents before the introduction of antibiotics [2]. Prior to the discovery of antibiotics, the desperate need of materials with antibacterial properties was followed by the application of silver in sutures, postoperative inflammation and infection prevention, and treatment of battle wounds during World War I. Usage of antibiotics, enzymes, metal ions, and quaternary ammonium compounds poses disadvantages, including the development of drug resistance and detrimental effects regarding the environment [3]. Numerous theories of the antibacterial mechanism of silver have been suggested in the past as the interest in silver's ability to fight microorganisms grew. Silver atoms are known to attach to the sulfur in thiol of vital enzymes involved in biological processes including ion transport and transmembrane energy generation [4]. Silver acts as a reducing agent to catalyze the reaction between cellular oxygen and hydrogen of thiol groups resulting in disulfide bonds [5]. Modifications in vital cellular molecules can provoke alterations in the cellular structure causing malfunctions that may finally result in apoptosis or necrosis. Disruption of cell respiration is a sure-fire cause of cell death. A decrease in the expression of maltose transporter, succinyl-coenzyme A synthetase, 30S ribosomal subunit protein, and fructose bisphosphate aldolase was observed in the cells treated with a $900 \mathrm{ppb}$ silver ion solution [6]. The silver ions deactivate the $30 \mathrm{~S}$ ribosomal subunit by binding to it. The deactivation of the ribosome complex leads to the interference in the translation of proteins [6]. Succinyl-coenzyme A synthase, a crucial component of the TCA cycle, catalyzes the reaction that yields succinate from succinyl-CoA and simultaneously phosphorylates ADP to make ATP [7]. Disruption of glycolysis via silver is caused by the deactivation of fructose bisphosphate aldolase. Fructose bisphosphate aldolase catalyzes the cleavage of fructose-1,6-bisphosphate into glyceraldehyde 3-phosphate and dihydroxyacetone phosphate [7]. A maltose transporter, MalK, present in the cytoplasmic membrane is another vital organelle affected by the silver [8]. The disruption of any one of these processes can contribute to the mortality of bacterial cells. Other mechanisms regarding silver's mechanism of action concerns silver's intracellular interaction with differing cell components. Klueh et al. proposed that $\mathrm{Ag}^{+}$ions can enter cells and denature DNA molecules especially by disrupting the hydrogen bonds between purine and pyrimidine base pairs [4]. The presence of numerous possible disruptions of cell functions may explain silver's capability of inhibiting bacterial growth of various species. Silver is currently used in wound dressings [9], endotracheal tubes [10], surgical masks [11], cotton fibers [12], drinking water purification [13], antibacterial glass [7], food packaging [14], clothing, burn wound-care cream, and many other everyday applications. The antibacterial properties of silver are only exhibited in its ionic form. It has been reported that nonionized silver from an insoluble silver source is unable to inhibit the growth of bacteria [15]. Among silver salts, silver sulfadiazine (AgSD) is commonly used as a source of silver ions and is known as a broad-spectrum antimicrobial substance [16]. Using its wide range of effectiveness against bacteria, silver sulfadiazine has been widely used in burn wound-care. Topical cream containing the $\mathrm{AgSD}$ is currently commercially available. In many studies regarding the antibacterial properties of silver and silver nanoparticles, silver sulfadiazine is considered the gold standard and is used to compare the potency of the bactericidal activity of novel substances [17]. By first understanding the mechanism of action of the antibacterial activity of silver at a molecular level, the compound can be improved upon by not only decreasing its size, but also by carrying out modifications that result in synergism.

Nanoparticles are categorized as particles with at least one dimension between 1 and $100 \mathrm{~nm}$ [18]. These particles are considered as the bridge between bulk materials and atomic structures due to their size. The synthesis of nanocomposites requires a stabilizing agent that keeps the substance from agglomerating as a result of attractive van der Waals forces. The range of applications for nanoparticles is immense due to its versatility in functionality. Varying the synthesis method of nanoparticles, type of stabilizers and reducing agents used during the synthesis vastly affects the properties of the product. Silver nanoparticles can be synthesized by methods such as evaporation-condensation [19], laser ablation [20], chemical reduction [21], and microemulsion [22]. Certain physical parameters, including particle size, shape, and size distribution, alter the properties and potencies of the nanoparticles. Thus, the nanoscale of the particles may cause the rise of unforeseen characteristics exhibited in the same compound in bulk. These unique properties of 
nanoparticles are the basis of its broad application in modern technology and science. Nanoparticle-unique properties include large surface area-to-volume ratio, uniformity, suspension formation, quantum confinement [23], and surface plasmon resonance [24]. One of the most utilized properties of the nanoparticles is its large surface area-to-volume ratio. The nano-silver's large surface area has the effect of increasing the expression of its properties. For example, the antibacterial activity of silver increases in nanoparticles, due to the increase in the contact area between the cells and the substance allowing more silver ions to enter the cell. The driving force for diffusion, which significantly elevates the activity of its properties, is a product of the high surface area-to-volume ratio. Surface plasmon resonance is used to characterize many synthesized nanoparticles via UV-Vis spectrometry as its presence confirms the formation of the nanoparticles without aggregation [25, 26]. Additionally, silver nanoparticles can be characterized through scanning electron microscopy (SEM), atomic force microscopy (AFM), dynamic light scattering (DLS), transmission electron microscopy (TEM), Fourier transform infrared spectroscopy, X-ray photoelectron spectroscopy (XPS), and powder X-ray diffractometry (XRD) [27]. Like other nanoparticles, the particles' tendency to agglomerate is one of the major obstacles of silver nanoparticle formation. Furthermore, in the biological experimental aspect of the silver nanoparticles, the agglomeration of the AgNPs in biological media such as DMEM tends to increase, making it difficult to accurately study the concentration dependent effects of the AgNPs [28]. Thus, it is important to select a ligand that is physically suitable in preventing the agglomeration of the silver ions at the nano-level by acting as a stabilizing agent. Pokhrel et al. reports that certain organic ligands with the ability to receive waters are able to differentially modify the surface of silver nanoparticles and alter their environmental persistence [29]. Silver nanoparticles are stabilized electrostatically, sterically, and electrosterically [30]. The conjugation of silver nanoparticles at its surface broadens the horizon of applications since the properties of the conjugation material are maintained after the reaction. Thus, as long as the conjugation is successful without any changes in the molecular structure, the conjugated silver nanoparticles should be able to exhibit the desired properties of the ligands.

This review of silver nanoparticles offers a unique indepth overview of the antibacterial silver nanoparticles, specifically in the aspects of parameter-dependent antibacterial potency, methods of enhancing the biocidal efficacy, and utilization of functionalized silver nanoparticles as wound treatment systems. By summarizing and integrating the parameter dependence of AgNPs' antibacterial activities provides the readers of the general overview of the parameter specific trend of the properties of the particles, allowing them to synthesize silver nanoparticles with the desired specifications for further research. The various methods of enhancing the antimicrobial properties of the AgNPs presents diverse methods of modifying silver nanoparticles to further aid in inhibiting bacterial growth. By collecting the highlighted works in this field, this review may allow scientists to apply specific alterations to their own work for better results. The marketable bi-functionality of wound-healing antibacterial silver nanoparticles has been the driving point of the usage of AgNPs in the field. The conjugated silver nanoparticles are discussed in detail regarding its preparation and woundhealing promotion properties. The wound-healing promotion properties in this review were focused on the materials' multifunctionality to stimulate cell proliferation and inhibit bacterial growth, which differentiates this paper from other reviews about silver nanoparticles.

\section{Parameter-dependent silver nanoparticle antibacterial properties}

The antibacterial activity of silver nanoparticles is influenced by the dimensions of the nanoparticles (e.g., size and shape). Generally, smaller particles exhibit greater antimicrobial effect [31]. The antibacterial effect of silver nanoparticles in the size range of $1-100 \mathrm{~nm}$ on Gramnegative bacteria was studied using commercially available nanoparticle powders [32]. The antibacterial effect of silver nanoparticles was studied with different concentrations of prepared nano-silver $(0,25,50,75,100 \mu \mathrm{g} / \mathrm{ml})$ inoculated with $10 \mathrm{ml}$ of an $E$. coli bacterial culture. The interactions between bacteria and nanoparticles were analyzed by the measurement of the optical density at $595 \mathrm{~nm}$. The electrochemical nature of silver nanoparticles was analyzed by stripping voltammetry. The size of silver nanoparticles was confirmed by TEM to be in the range of $1-10 \mathrm{~nm}$. A statistical study and HRTEM study confirmed that $98 \%$ of the particles with diameters between 1 and $10 \mathrm{~nm}$ were different in shapes. The study on the effect of different concentrations of silver nanoparticles on the growth of bacteria demonstrated that at concentrations above $75 \mu \mathrm{g} /$ $\mathrm{ml}$ no significant bacterial growth occurred. Additionally, the presence of silver nanoparticles in the cell membrane and cytosol of the bacteria was confirmed by TEM. Based on the observation of the TEM and HAADF images, the smaller the particles size $(5 \mathrm{~nm})$ the higher the antibacterial activity of the silver nanoparticles. Panacek et al. reported a simple one pot synthesis of colloid AgNPs with different sizes (Tollens process) [33]. In this method, four different saccharides, glucose, galactose, maltose and lactose, were used as reducing agents. The size of the silver colloid 
particles ranged from 25 to $100 \mathrm{~nm}$ on average. The synthesized particles were characterized by DLS, TEM and UV-visible absorption spectrometry. The reduction of diamino silver ions by maltose produced silver colloid nanoparticles with an average size of $25 \mathrm{~nm}$. Through MIC and MBC determination, it was found that the smallest silver colloid particles size at $25 \mathrm{~nm}$ showed the greatest bacterial termination and inhibition against Gram-positive and Gram-negative bacteria including highly multi-resistant strains such as methicillin-resistant Staphylococcus aureus (MRSA). Martinez-Castanon et al. investigated the synthesis of silver nanoparticles with different sizes (7, 29 and $89 \mathrm{~nm}$ ) [34]. This group used gallic acid as a reducing as well as stabilizing agent. Through TEM analysis, the silver nanoparticles were determined to have spherical (7 and $29 \mathrm{~nm}$ ) and pseudospherical shapes (89 nm). In order to produce small-sized silver nanoparticles (7 and $29 \mathrm{~nm})$, this group performed a reduction reaction at $\mathrm{pH} 11$ and 10 , respectively. This group used a UV light $(254 \mathrm{~nm}, 25 \mathrm{~W})$ irradiation method for $30 \mathrm{~min}$ and heated for $30 \mathrm{~min}$ at $80{ }^{\circ} \mathrm{C}$ to obtain $89 \mathrm{~nm}$ silver nanoparticles. The antibacterial activities of the products were tested against Gramnegative bacteria (E. coli) and Gram-positive bacteria $(S$. aureus). The $7 \mathrm{~nm}$ silver nanoparticles exhibited the best antibacterial activity against both species. Because of the miniscule nanoparticles size $(7 \mathrm{~nm})$ and high surface area, silver nanoparticles with $7 \mathrm{~nm}$ were able to reach the nuclear content of bacteria efficiently; therefore being able to kill bacteria more effectively. Espinosa-Cristóbal et al. studied the antibacterial effect of silver nanoparticles against Streptococcus mutans, the microorganism associated with dental caries [35]. The three differently sized AgNPs ( 8.416 .1 , and $98 \mathrm{~nm}$ ) were prepared by using a modified reaction condition of that reported by MartinezCastanon et al. The morphology of silver nanoparticle with $S$. mutans treatment was observed using atomic force microscopy (AFM). The nanoparticles with an average diameter of $8.4 \mathrm{~nm}$ showed better antibacterial activity compared with those of the larger nanoparticles (16.1 and $98 \mathrm{~nm}$ ). Espinosa-Cristóbal et al. suggested that smaller nanoparticles release more $\mathrm{Ag}^{+}$ions, thus causing an increase in their antibacterial effect. Lu et al. reported on the size-dependent antibacterial activities of AgNPs against oral anaerobic pathogenic bacteria [36]. In this work, three silver nanoparticles with different sizes $(\sim 5,15$ and $55 \mathrm{~nm}$ ) were prepared by using two methods. To obtain the smaller nanoparticles, $\mathrm{NaBH}_{4}$ was used as a reducing agent and the larger set of particles was synthesized by PEG reduction. The bacterial inhibition study was evaluated by colony counting assays, growth inhibition curve method, and corresponding MIC measurements against five anaerobic oral pathogenic bacteria Actinobacillus actinomycetemcomitans, Fusobacterium nucleatum,
Streptococcus mutans, Streptococcus sanguis, Streptococcus mitis and aerobic bacteria E. coli. The antibacterial effects of the particles against anaerobic bacteria were lower than those against aerobic bacteria. Thus, it can be inferred that the aerobic bacteria were more susceptible to AgNPs than the anaerobic oral pathogenic bacteria. The $5 \mathrm{~nm}$ AgNPs presented the best antibacterial activity against $E$. coli and $F$. nucleatum. Considering the higher antimicrobial properties and lower toxicity of AgNPs compared to those of Ag compounds, such as silver nitrate and silver sulfadiazine, they could be used for the treatment of periodontal pathogens with greater benefits. Recently, Agnihotri et al. reported the complete and detailed investigation for size-specific antibacterial efficacy of AgNPs [37]. The AgNPs of different sizes $(5,7,10,15,20,30,50$, 63,85 and $100 \mathrm{~nm}$ ) were synthesized using sodium borohydride as a primary reductant and trisodium citrate as a reductant as well as a stabilizing agent. The synthesis of nanoparticles was based on the co-reduction approach using a two-stage thermal treatment as shown in Fig. 1. From the results, the antibacterial efficacies of the AgNPs with $>10 \mathrm{~nm}$ size were significantly improved as confirmed through delayed bacterial growth kinetics, corresponding MIC/MBC values, and disk diffusion tests. The AgNPs with an average diameter of $5 \mathrm{~nm}$ showed more potent bactericidal activity against all the tested strains [(Gram-negative (E. coli MTCC 443, E. coli MTCC 739) and Gram-positive (B. subtilis MTCC 441, and S. aureus NCIM5021)] compared to AgNPs with 7 and $10 \mathrm{~nm}$ diameters at the same bacterial concentrations. In conclusion, the smallest AgNPs (5 and $10 \mathrm{~nm}$ ) showed improved biocidal activity towards many different microbial strains.

Song et al. investigated the shape-dependent antibacterial properties of silver nanoparticles [38]. There are many literatures suggesting the size dependency of the antibacterial activity of the nano-silver, but Song's group provides a novel angle of the shape dependency of the AgNPs' bactericidal capabilities. The silver nanoparticles with different shapes were prepared by seeded growth method (spherical) and solution phase method (rod and triangle shape). The antibacterial activity of AgNPs with different shapes was tested against Gram-negative bacteria E. coli. The silver nanoparticles were characterized by UV-visible spectroscopy and energy filtering transmission microscopy (EFTEM). The UV-visible spectra and TEM images confirmed the spherical shape of the nanoparticles. The EFTEM images (as shown in Fig. 1) for the rod and the truncated triangle AgNPs were further confirmed by OPML-XRD pattern and TEM. The bacterial killing kinetics of the AgNPs were examined and determined by absorbance measurements at $600 \mathrm{~nm}$. The total silver concentration content was quantified by ICP-MS. The silver content at $12.5 \mu \mathrm{g}$ and above $(50$ and $100 \mu \mathrm{g}$ ) showed 


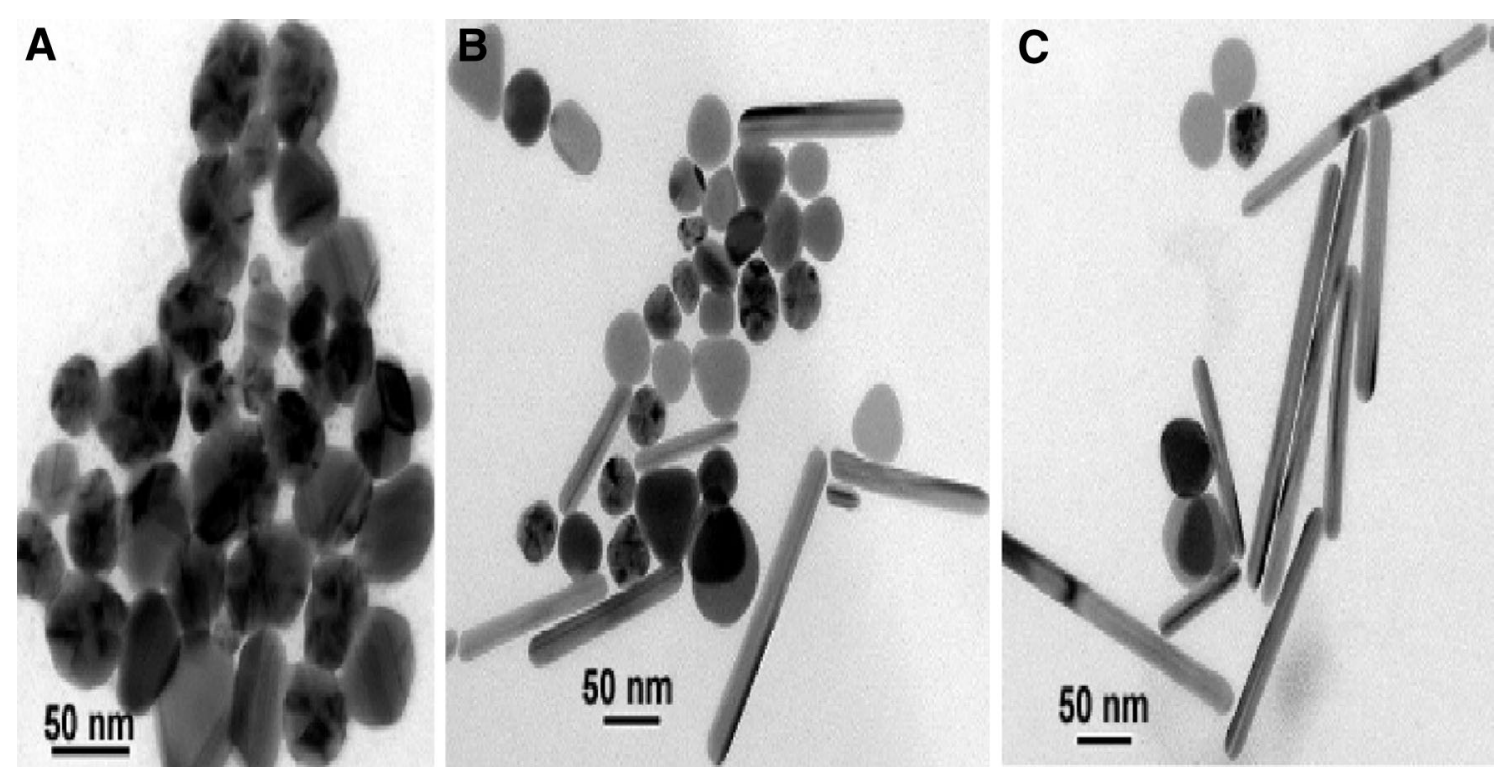

Fig. 1 EFTEM images of silver nanoparticles. a Spherical nanoparticles synthesized by citrate reduction. b Silver nanoparticles of different shapes. c Purified rod-shaped nanoparticles [38]. Reproduced by permission of The American Society for Microbiology

$100 \%$ bacterial inhibition in spherical nanoparticles. Full bacterial inhibition at $10 \mu \mathrm{g}$ of silver content was observed for truncated triangular AgNPs. Compared to the other shapes of silver nanoparticles, the truncated triangular AgNPs were the most effective in preventing bacterial growth. This phenomenon was explained by the percent active facet of the various AgNPs. The high percent composition of [111] facet of the top basal plane of the truncated triangular AgNPs observed via the OPML-XRD results provide insight into the truncated nanoplates' highatomic density structure, which is understood to be the major contributing factor in its enhanced bactericidal efficacy. For comparison, pentagonal rod-shaped and spherical silver nanoparticles exhibited a lower percentage of [100] facet and lower antibacterial activities. By association, the high-atom-density structures seem to be responsible for the increase in bacterial growth inhibition, possibly due to the increase in the level of interaction between the reactive silver ions and the bacterial membrane at an atomic level (Fig. 2).

The use of crown ether solution is a different approach in silver nanoparticle preparation. Berdnikova et al. utilizes a crown ether-mediated photochemical reduction, a synthetic technic advantageous in both spatial and temporal control, of silver ions in silver nanoparticle formation [39]. In this study, a mixture of cationic DNA intercalator, benzo[ $[b]$ quinolizinium-crown ether, and DNA was explored as a AgNP formation moiety in order to avoid undesired degradation as a result of the exposure to highenergy light needed for the AgNP synthesis, as shown in
Fig. 3. The crown-ether acted as a mediator between the DNA and the $\mathrm{Ag}^{+}$. The DNA intercalator acted as a light harvesting photosensitizer for the electron transfer reaction to initiate the process of the silver ion reduction. The high binding affinity of the crown ether to $\mathrm{Ag}^{+}$provides a close proximity between the different components of this ternary composite, which yields the following benefits: high efficiency in AgNP synthesis, formation of stable AgNPs, and improvement in the reproducibility of size. The utilization of crown ether in the AgNP formation process has been researched quite extensively, but not much in the direction of its antibacterial activity. Due to the variation in the synthesis step, the products may exhibit a different level of antibacterial activity. The ability to enhance the control of the AgNP parameters may be contributed to the physical binding of the silver ions at a molecular level. In another study, crown ether was embedded in a polymer, poly(styrene), in order to provide a more flexible binding structure for the silver nanoparticles [40]. The modifications of the crown ethers seem to be the deciding factor in the various silver nanoparticle mediating molecules. Although efficient and easy to operate, crown ethers' presence in the silver nanoparticle sample will significantly increase its cytotoxicity not only against bacteria, but also against healthy cells. Furthering the research of the crown ethers and increasing its biocompatibility via enhanced purification or modification of the crown ether itself, seems necessary in the field in order for the crown ether synthesized AgNPs to be utilized in wound treatment. Aside from the bactericidal properties of the crown ether silver 


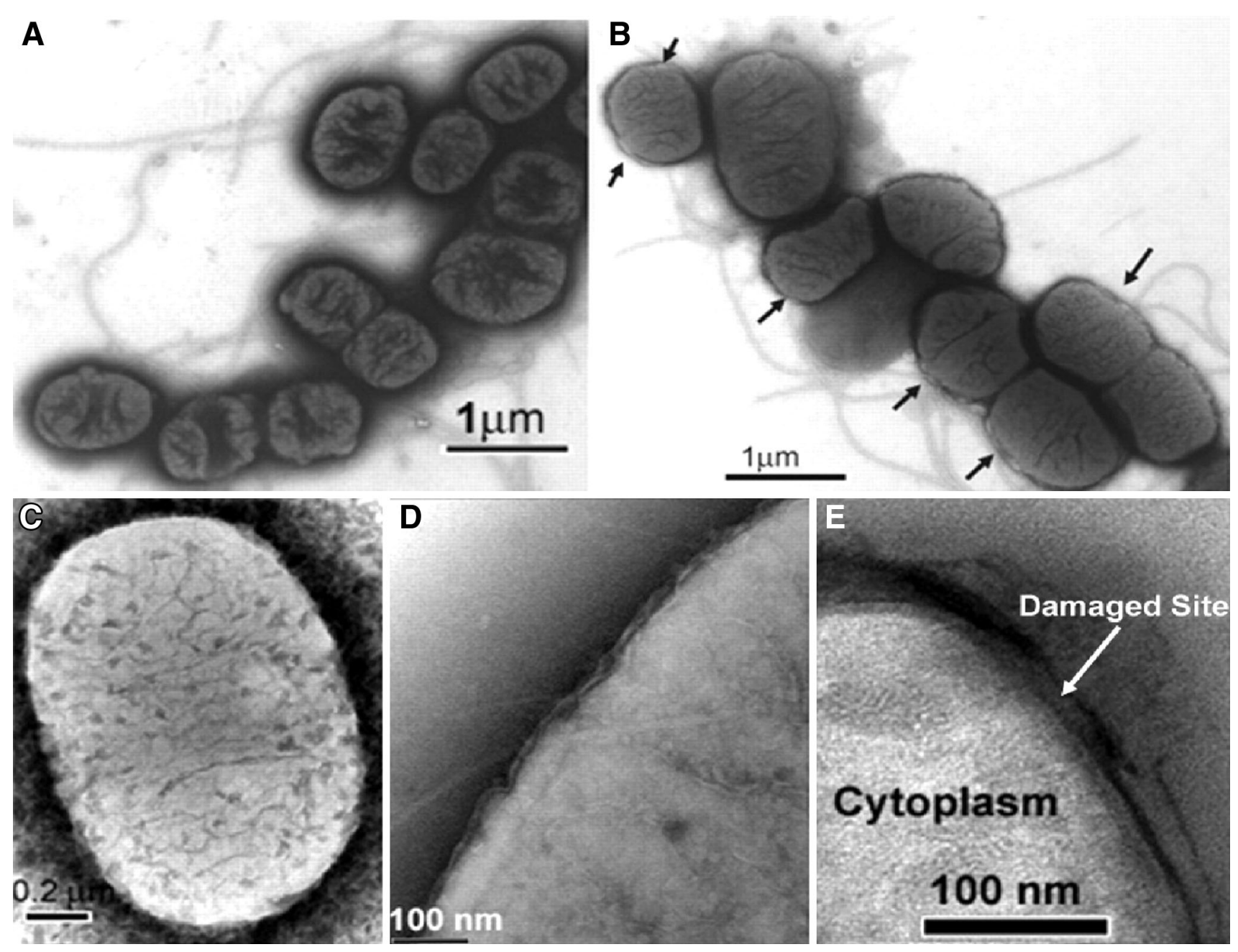

Fig. 2 EFTEM images of E. coli cells. a Untreated E. coli. Flagella can be seen. b E. coli grown on agar plates supplemented with $\mathrm{Ag}^{+}$ $\left(\mathrm{AgNO}_{3}\right)$. Arrows indicate partially damaged membranes. These cells are viable. c E. coli treated with triangular silver nanoplates. Silver nanoparticles appear as dark irregular pits on the cell surface. d $E$. coli treated with spherical silver nanoparticles. e Enlarged image of part of the bacterial cell membrane treated with triangular silver nanoparticles. The cell membrane is damaged at multiple locations [38]. Reproduced by permission of The American Society for Microbiology

Fig. 3 A schematic

representation of the

photoinduced reduction of $\mathrm{Ag}^{+}$ by DNA-bound

benzoquinolizinium 1a [39].

Reproduced by permission of

The Royal Society of Chemistry (RSC) on behalf of the Centre

National de la Recherche

Scientifique (CNRS) and the RSC<smiles>[R]c1ccc2c[n+]3ccccc3cc2c1</smiles>

$1 a, b$<smiles>[Y10]C=CN1CCSCCOCCOCCSCC1</smiles>

1b: $R=$ NEt $_{2}$

$1 \mathrm{a}+\mathrm{Ag}^{+}+\mathrm{DNA}$

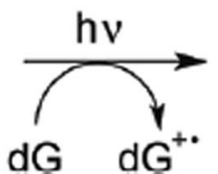

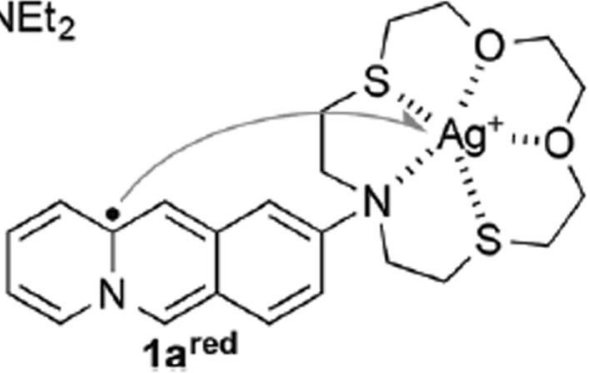


nanoparticles, the material has been used in colorimetric detection of metal ions with superb sensitivity. The colorimetric detection of AgNPs utilizes its ability to recognize metal ions and alter the solution's optical properties by aggregation, thus simplifying the detection method [41]. Ratnarathorn et al. developed a simple AgNP-bound paperbased copper colorimetric sensing devices [42]. These devices allowed the users to rapidly and easily detect the presence of copper in solutions with a detection limit of $7.8 \mathrm{nM}$ with the naked eye. The speed, ease of operation, low detection limit, and mobility of the paper devices make this colorimetric detector useful and marketable. Unlike most metal ions, the detection of $\mathrm{Pb}^{2+}$ ions were not readily detected by ACE-AgNP based colorimetric methods. In order to overcome the limitations of detecting lead ions, dynamic light scattering was used to detect $\mathrm{Pb}^{2+}$ ions with ultra-sensitivity using aza-crown-ether modified AgNPs [43]. The use of nanoparticles' unique plasmon resonance allows for the sensitive detection of various metal ions in solution, which is ideal in the detection of such substances in environmental field studies of water.

\section{Enhancing the bactericidal efficiency of AgNPs}

The antimicrobial property of silver nanoparticles is one of the most useful aspects of the material. There has been a great deal of research in improving the efficacy of bacterial inhibition from silver nanoparticles. The improvement in the antibacterial efficacy is essential as silver nanoparticles do exhibit toxicity at levels above the threshold concentration. Hence, a material that exhibits the same amount of antibacterial activity at a lower concentration may be necessary to decrease the possibility of causing unwanted damage in the cells. Researchers have studied, improved, and developed a variety of methods to synthesize novel silver nanoparticles. The bacteria killing activities of the products were tested by treating different kinds of bacteria with the materials and monitoring the cells. Reports of novel synthetic methods leading to greater bactericidal activity have been prevalent in the field of nanotechnology.

Silver nanoparticles prepared by using a blend of reducing agents D-glucose and hydrazine were better able to limit the growth of bacteria [44]. The stability of the nanoparticles was tested over a period of 4 weeks to ensure their long-term molecular stability. The blend-reduced silver nanoparticles were tested on $S$. aureus and ampicillin-resistant $S$. aureus on liquid broth, inoculation, and agar plates. This paper also reported that the antibiotic resistance to ampicillin of the bacteria was not a factor in hindering the antibacterial inhibition of the prepared nanoparticles. Furthermore, Dr. Shrivastava's group reported a more specific mechanism that may be responsible for silver's antibacterial property; the silver nanoparticles anchored to and penetrated the cell wall of bacteria, then modulated its signaling by dephosphorylating major peptide substrates of tyrosine residues [44]. This mechanism was supported by the observation of noticeable dephosphorylation in two peptides of E. coli and S. typhi. Tyrosine phosphorylation is responsible for activating UDP-glucose dehydrogenases and RNA polymerase sigma factors [45]. Dephosphorylation of the peptides may suggest the inhibition of the enzymes and critically impact the growth of bacteria [45]. Understanding and utilizing the bacterial signal transduction disruption of silver nanoparticles will aid in the development of highly efficient antibiotic nanomaterials. Certain studies took a simple, but novel approach of enhancing the antibiotic activity of silver nanoparticles by forming combinations of antibiotics and silver nanoparticles. Combining antibiotics and capped silver nanoparticles seemed to synergistically augment the antibacterial activities of both components. Dr. Kora and

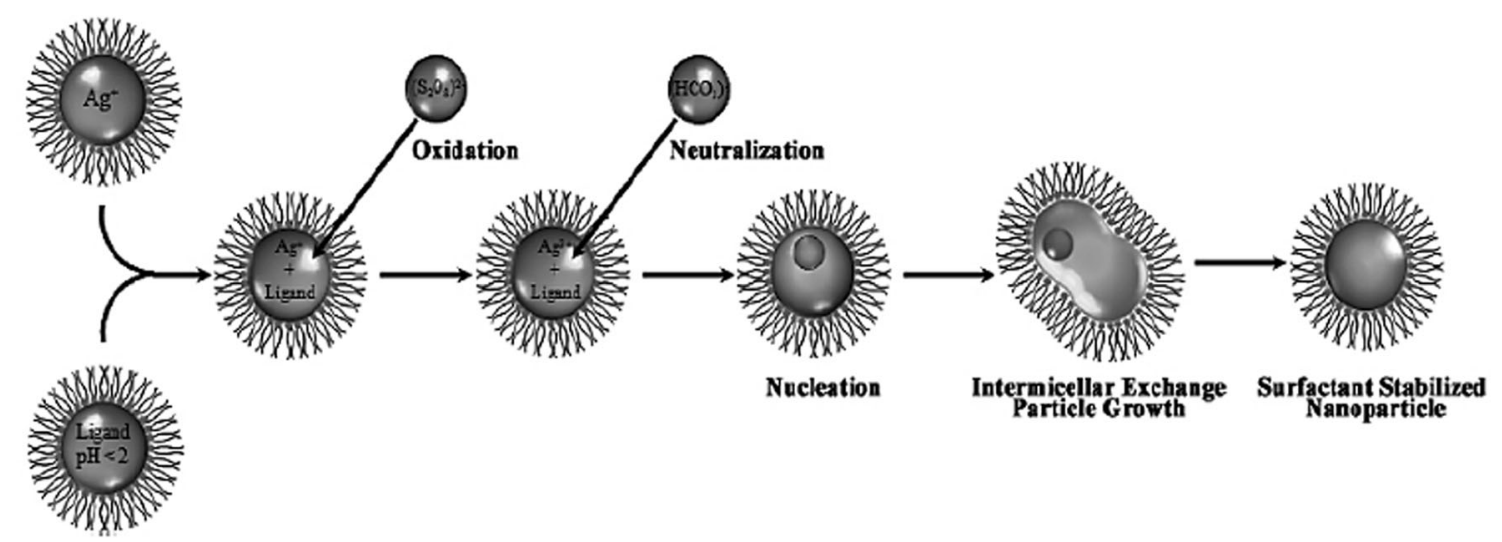

Fig. 4 The synthesis sequence for silver chlorhexidine nanoparticles in a reverse microemulsion. Reprinted with permission from Pal et al. [17]. Copyright 2009 American Chemical Society 
Dr. Rastogi reported the levels of enhancement in antibacterial activity from various combinations of silver nanoparticles and antibiotics. According to the study, the different combinations of sodium dodecyl sulfate (SDS) capped and polyvinylpyrrolidone (PVP) capped silver nanoparticles with ampicillin, tetracycline, and streptomycin all resulted in enhancements in their antibiotic activities compared to that of the individual components, when tested on S. aureus and E. coli [46]. The PVP-capped silver nanoparticles exhibited the greatest enhancement in bactericidal activity, especially in association with streptomycin on S. aureus and ampicillin on E. coli, at 45.4 and $50.0 \%$, respectively. The silver nanoparticles showed promising results in enhancing the antibiotic efficacy of already established antibiotics. Further research in this area may lead to discoveries in combinations that induce additionally enhanced antibacterial activities. Also diversifying the components of the combinations may allow for improvements in efficacy by attacking the bacteria from different approaches simultaneously. Polymer micelles embedded with silver nanoparticles showed strong bactericidal activity. Selective hydrolysis of poly(vinyl acetate)$b$-poly(acrylonitrile) copolymer, synthesized through a radical polymerization, resulted in a new amphiphilic poly(vinyl alcohol)- $b$-poly(acrylonitrile) [47]. The amphiphilic block copolymer product was used as a nanoreactor to form silver nanoparticle-embedded micelles by selfassembly. The micelles presented strong bactericidal effects on E. coli, S. aureus, P. aeruginosa, and spore solutions of Bacillus subtilis [47]. Creating a nanocomposite consisted of different structures is a more complex, but effective way of increasing silver nanoparticles' efficacy in preventing the growth of bacteria. Liang's group reported the development of a sandwich-like antibacterial reagent that proved to be more effective than simple silver nanoparticles [3]. AgNPs/halloysite nanotubes/graphene nanocomposites (Ag/HNTs/rGO), sandwich-shaped reagents from the study, consisted of multiple parts: halloysite nanotubes, silver nanoparticles, graphene oxide nanosheets, and DOPA or L-3,4-dihydroxyphenylalanine. According to the paper, the reagent was formed by a single step method of forming silver nanoparticles by reducing DOPA at the GO nanosheets or surface of HNTs. DOPA acts as an adhesive for all the components keeping the structure together by utilizing its adhesion properties. The group confirms the formation of the nanocomposites through transmission electron microscopy (TEM), HRTEM, energy dispersive spectrum (EDS), and FTIR. The increase in surface area was measured using the adsorption of $\mathrm{N}_{2}$. E. coli and $S$. aureus was used as the bacteria models in the measurement of the Ag/HNTs/rGOs antimicrobial activity. The bactericidal efficacy of the synthesized product was compared to that of the starting materials: graphene oxide, silver, silver nanoparticle packed halloysite nanotubes, and silver nanoparticles attached to graphene oxide nanosheets. The novel sandwich-shaped nanocomposites exhibited the lowest MIC among the various test groups at $2 \mu \mathrm{g} / \mathrm{ml}$ [3]. The effect of the different oxidation states of silver was studied by forming stable silver complexes with silver oxidation states 1, 2, and 3 [48]. Silver (I) chlorhexidine was prepared through precipitating the complex in situ in a weakly acidic aqueous solution of $\mathrm{AgNO}_{3}$. The oxidation of $\mathrm{Ag}$ (I) followed by the complexation with chlorhexidine yielded silver (II) chlorhexidine. The antibacterial activities of both materials were measured by the determination of MICs and MBCs on Acinetobacter calcoaceticus, Citrobacter freundii, E. faecalis, Klebsiella pneumonia, P. acnes, Pseudomonas aeruginosa, S. epidermidis, and S. aureus. The higher valent $\mathrm{Ag}(\mathrm{II}) \mathrm{CHX}$ exhibited the lowest MIC and $\mathrm{MBC}$ in all bacteria. The killing kinetics of the compound were also monitored through monitoring the compoundtreated bacteria culture as a variable of time. Overall, the synthesized AgCHX compounds were able to reach its bactericidal endpoint, which was designated as the presence of a decrease in the level of $\mathrm{cfu} / \mathrm{mL}$ equal to or $>99.9 \%$, much faster than $\mathrm{CHX}, \mathrm{AgNO}_{3}$, and silver sulfadiazine. Trivalent silver polydiguanide complex nanocomposites were synthesized via oxidation of silver (I) followed by a reverse microemulsion complexation reaction using a polydiguanide, as shown in Fig. 4 [17]. The antibacterial activity of trivalent $\mathrm{Ag}$ (III)CHX was also tested in the same 8 bacteria species as $\mathrm{Ag}(\mathrm{II}) \mathrm{CHX}$ and $\mathrm{Ag}(\mathrm{I}) \mathrm{CHX}$. The trivalent silver nanoparticles exhibited better antibacterial activities compared to those of chlorhexidine, $\mathrm{AgNO}_{3}$, and silver sulfadiazine. These results confirm the strengthening of the bactericidal activity of nano-silver produced by the high valency of silver.

\section{Wound-healing properties of conjugated silver nanoparticles}

Wound-healing is a complex biological process that occurs as a mean of maintaining the human body. It undergoes various processes that include: cell proliferation, inflammation, migration, angiogenesis and extracellular matrix (ECM) production [49]. Initially, neutrophils, monocytes, and lymphocytes surround the wounded area. In the proliferation phase endothelial cells, fibroblasts, and other types of cells gather in the wounded area and start to reproduce, release matrix materials, and form functional capillaries and layers of epithelial cells that spread over the open wound. In the final phase, matrix materials and collagen are secreted and remodeled to fix tissue strength. 
Silver and silver-related materials such as silver sulfadiazine, have been well explored as topical agents for wound treatment. These antibiotic compounds are prone to cause antibiotic resistance or inhibition during the woundhealing process. For instance, silver sulfadiazine increases wound epithelialization but impairs wound contraction, which is a vital part of the repair process that pulls the wound edges together to reduce the defect [50]. Similarly, antibiotic sulfamylon demonstrated decreased keratinocyte growth rates [51]. In addition to this, other antibiotic agents in high concentrations produce injurious effects on endothelial and fibroblasts cells [52]. Due to all the reasons presented, the development of a novel and more effective system of antibacterial/proliferative wound treatment will prove beneficial in improving the quality of wound treatment.

A large number of silver-based dressings is currently available in the market. Silver in the form of impregnated bandages or as topical creams are used as wound treatment agents. Also, it is used in the form of fibers, polymeric scaffolds impregnated and coated with a silver salt or in a nano-particulate form. The silver-based dressings exhibit fast and broad-spectrum bactericidal activity against various pathogenic bacteria species. However, there are not many reports available on the direct wound-healing mechanism by silver or silver nanoparticles. Recently, a few research groups have developed novel silver-based materials such as scaffolds, composites and hydrogels that combine the killing of microbes and healing of wounds that may be useful in the field of wound repair systems.

In a recent study by Heo et al., a blend of natural and synthetic polymers containing nanofiber scaffolds and gelatin was prepared by electrospinning [53]. Along with the blended polymer solution, silver sulfadiazine (SSD) was mixed in and introduced into the nanofibers after the electrospinning method. The nanofiber scaffolds containing SSD served as a substrate for skin repair, but also delivered the chemical of interest in a controlled manner to stimulate regeneration. The controlled release of the drug was capable of controlling the reproduction of a wide array of bacteria and promoting damage restoration through stimulation and the prevention of infections leading to the enhancement of the burn-wound closure rate.

During the wound repair process, maintaining adequate moisture levels by avoiding dehydration around the wound area is critical. For this reason, hydrogels are preferred since they are able to maintain a moist environment at the wounded site, act as a barrier to microorganisms, and serve as a superior biocompatible material towards wound healing. Zengjie et al. have developed a wound dressing material comprising a $\mathrm{Ag} / \mathrm{graphene}$ polymer hydrogel for the effective killing of bacteria and the acceleration of wound healing [54]. Various hydrogels were synthesized by the crosslinking of Ag/graphene composites with $N, N^{\prime}$ methylene bisacrylamide and acrylic acid at different concentrations. Hydrogels prepared with the optimal graphene to silver mass ratio of 1:5 exhibited stronger antibacterial abilities compared to those of other hydrogels. Also, the optimal hydrogel exhibited various desirable properties including good biocompatibility, high swelling ratio, and high extensibility. Moreover, the efficacy of the product was tested in vivo on rats to check its healing potency. A histological examination of the material revealed that the hydrogel assists the reconstruction and thickening of the epidermis during the 15-day period of wound repair. Thus, multifunctional composites like graphene/silver hydrogels exhibited excellent antibacterial and wound closure properties for the development a novel wound dressing.

Song et al. developed multifunctional composites with both antibacterial and wound-healing properties [55]. In this work, a multifunctional therapeutic agent consisting of a wound-healing polypeptide histatin-1 (Hst) and a highly bactericidal agent silver(II)chlorhexidine complex, $\mathrm{Ag}(\mathrm{II}) \mathrm{CHX}$, was developed. $\mathrm{Ag}(\mathrm{II}) \mathrm{CHX}$ was first synthesized on a large scale then combined with the woundhealing polypeptide. These composites showed higher bactericidal activity against various pathogens.

Cell spreading is a very important component of the reepithelialization phase. Histatins are polypeptides isolated from human saliva. These peptides were studied for the in vitro epithelial cell migration-inducing properties of saliva [56]. Histatins also enhanced reepithelialization by inducing migration and spreading of cells. Histatin-1 (Hst1) and Histatin-2 (Hst-2) (12-38 Hst-1) were used as potent wound closure peptides, whereas Histatin-5 (Hst-5), another antimicrobial peptide of the histatin family, failed to produce any wound closure properties. Therefore, the effect of $\mathrm{Ag}(\mathrm{II}) \mathrm{CHX}$ on the cell-spreading activity of Hst-1 was tested by comparing the samples with and without the presence of $\mathrm{Ag}(\mathrm{II}) \mathrm{CHX}$. The concentrations silver complexes were selected according to its MIC values $(0.12-3 \mu \mathrm{M})$. Interestingly, cell-spreading assay performed for silver complex showed no wound closure property as shown in Fig. 5a, but it also did not impair the cell-spreading activity of the Hst-1 (Fig. 5b). The dosedependent activity of a silver complex, Hst-1[Ag(II)CHX], on cell-spreading was determined by measuring the cellfree gap. The microscopy images showed that the silver complex did not increase cell-spreading as shown in Fig. 5a, but it also did not affect the cell proliferation promoting properties of the Hst-1 (Fig. 5b). Relative cellfree gap $\left(\mathrm{RG}_{t}\right)$ calculated at various time intervals, showed Hst-1-[Ag (II) CHX] conjugate had cell-spreading activity. The conjugate's activity was similar to that of Hst-1 

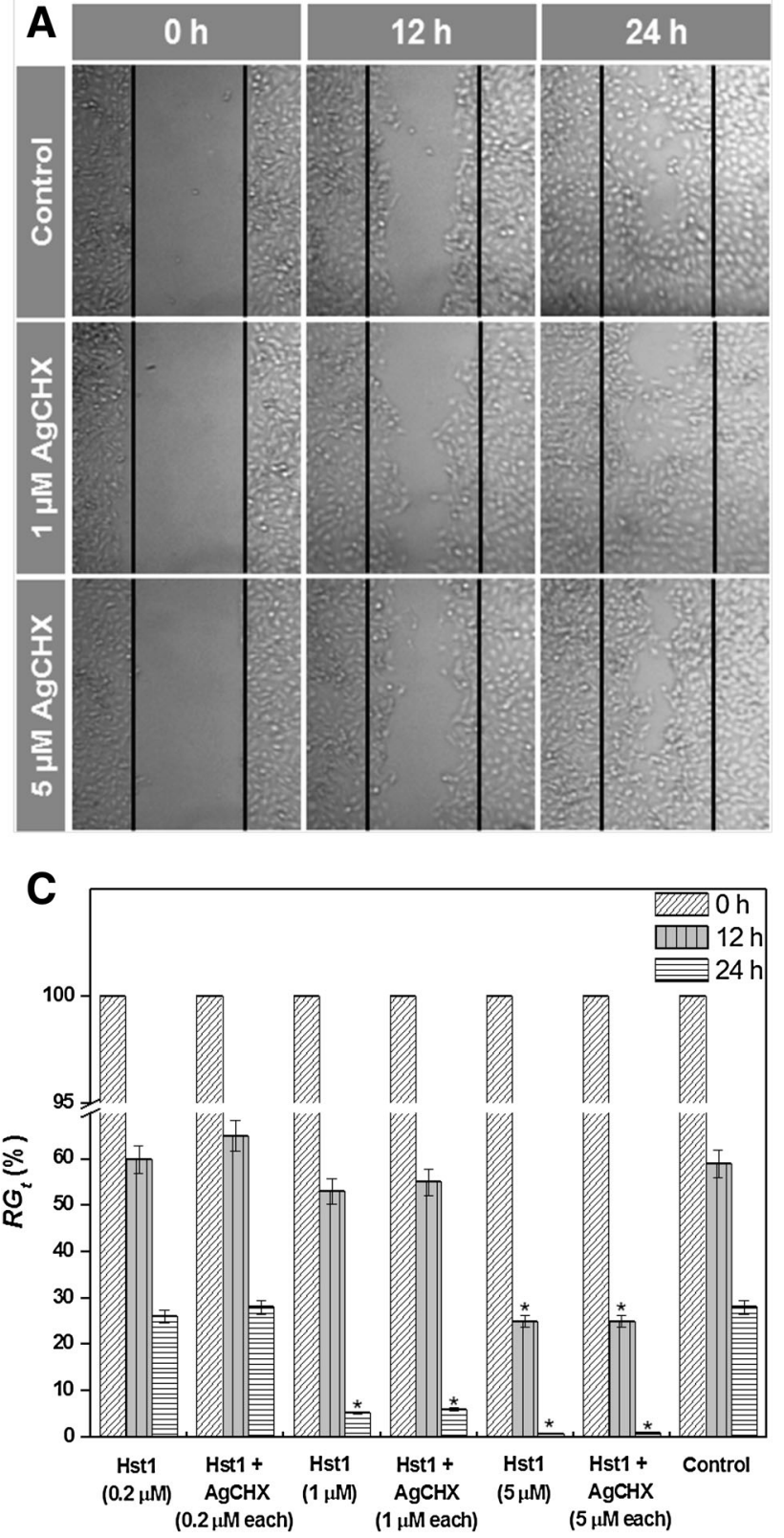

Fig. 5 Representative micrographs (a, b) and graphical representation of the results $(\mathbf{c}, \mathbf{d})$ of the in vitro cell-spreading assays. The cells were supplemented with different amounts of active agents and microscopic images were captured at different time intervals. The solid lines in images $(\mathbf{a}, \mathbf{b})$ represent the cell-free gap boundaries at $0 \mathrm{~h}$. The relative cell-free area $\left(\mathrm{RG}_{t}\right)(\mathbf{c})$, and the relative spreading

(Fig. 5c). The synthesized Hst-1, regardless of the AgCHX attachment, concentration dependently enhanced cellspreading. While relative cell-spreading (RGs) in the presence of $0.2 \mu \mathrm{M}$ Hst-1 was comparable to the control, higher concentrations of the peptide sharply increased the RGs for $12 \mathrm{~h}$ and complete cell coverage was achieved much faster than the control. Figure 5d demonstrates the
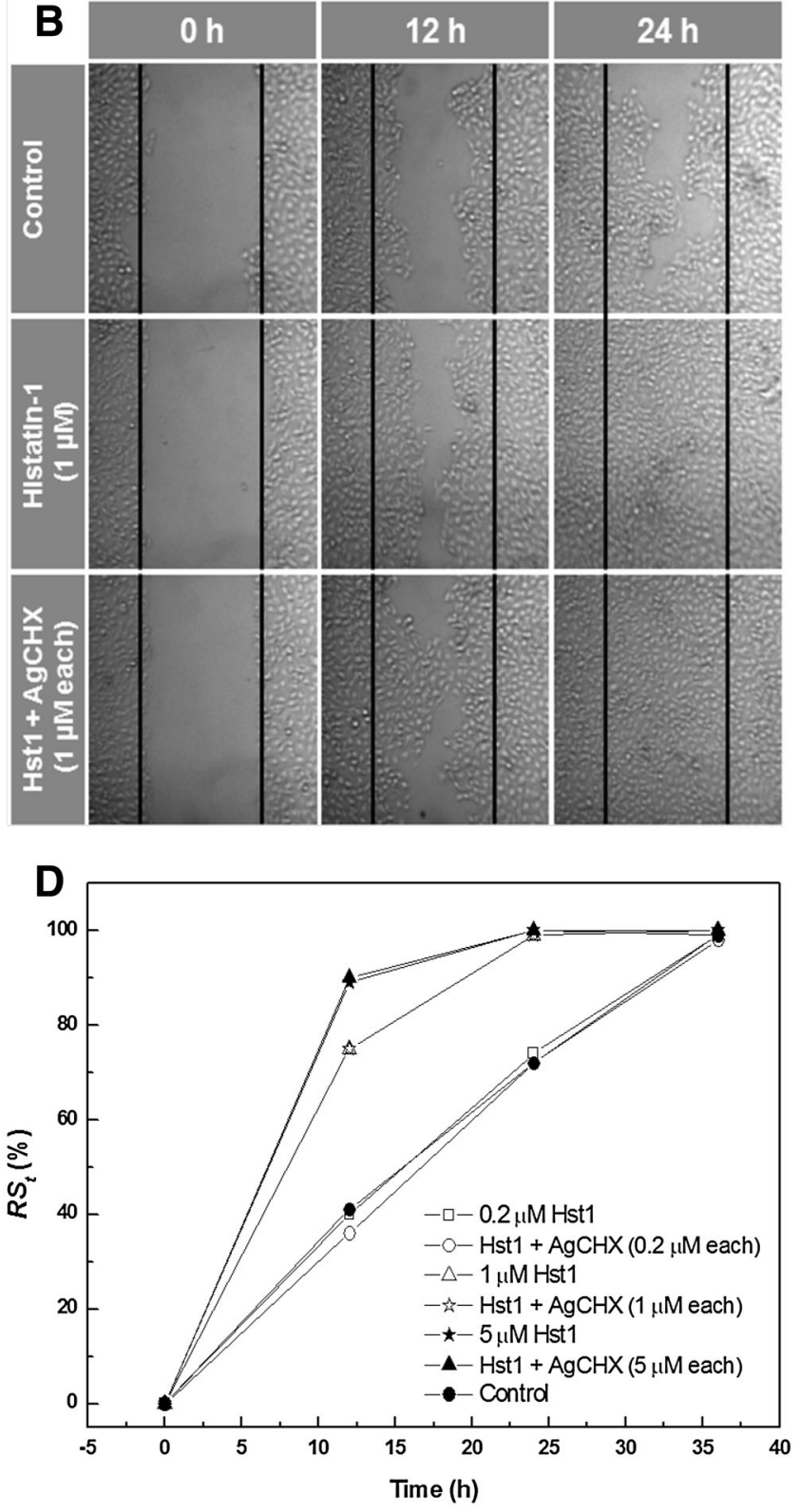

$\left(\mathrm{RS}_{t}\right)(\mathbf{d})$ at a given time $t$ were calculated by quantifying the cell-free area from the micrographs. The data represent mean $\pm \mathrm{SD} ; n=1$. $* P<0.01$ vs. appropriate control [55]. Reproduced by permission of The Royal Society of Chemistry (RSC) on behalf of the Centre National de la Recherche Scientifique (CNRS) and the RSC

increased level and rate of cell-spreading as a result of increased concentrations of Hst-1. The combination of antibacterial silver complex and wound-healing peptide presents a novel wound-healing system that aids in controlling and killing the bacteria around wound area and increase the proliferation during the wound-healing process. 


\section{Conclusion}

The versatility of silver nanoparticles is derived from its capability of modification. Size variation affects the potency of the antibacterial particles. Silver nanoparticles in different shapes also exhibit differing levels of antibacterial activities. The ease in the control of the size and shape of the nanoparticles grants the freedom in the manipulation of the particles as necessary. Incorporation of silver nanoparticles with pre-established antibiotics, variation in the synthetic method by using different reductants and stabilizers, conjugation of the silver nanoparticles with ligands that exhibit antibiotic activities themselves, formation of complex nanocomposites, and increasing the valency of the silver ion were all successful in enhancing the nanoparticles' ability to inhibit bacterial growth through various mechanisms, some of which are still unknown. Further stretching the utility of the silver nanoparticles, the particles were incorporated in several experimental wound treatment systems. By taking advantage of the antimicrobial activities of AgNPs in a variety of forms and cell proliferation activities of other materials, wound repair aid systems with better efficacy and safety can be developed in the future. With peptide conjugation capabilities of the nanoparticles, a whole new spectrum of applications was open as a field of modification research. The immense range of utility of the AgNPs comes with certain environmental and health safety responsibilities. Potential dangers of this material need to be studied along with the advancement of the nanoparticulates as those properties advantageous in the antibacterial applications may pose unprecedented environmental threats. This review presents the utilization, modification, and determination of antibacterial activity of many different types of silver nanoparticles exhibiting AgNPs' versatility. The versatile capabilities of Silver nanoparticles should be researched further to improve upon those applications that are being used today and to discover new applications of the scientifically promising silver nanoparticles. The green synthesis of the silver nanoparticles have been increasingly gaining interest for its reduction in the production of environmentally harmful byproducts and potentially increasing the AgNPs biocompatibility and decreasing its toxicity. Use of natural products such as biopolymers in the preparation of nanosilver seems promising in the development of medically applicable silver nanoparticles. Enhancing AgNP's biocompatibility, environmental sustainability in production, antibacterial activity and reducing its cytotoxicity and side effects should be considered as the major directions in antibacterial AgNP research.

Acknowledgments This research work was supported by the National Research Foundation of Korea (NRF) Grant funded by the Ministry of Education, Science and Technology (MEST)
(2015R1A2A1A05001842). We are grateful to the Research Institute of Pharmaceutical Sciences at Seoul National University for providing some experimental equipment.

\section{Compliance with ethical standards}

Conflict of interest None to declare.

Authors' contributions GWN contributed the Enhancing the Bactericidal Efficiency of AgNPs section of the article, wrote the introduction section and proofread the manuscript. SR contributed in the Wound Healing Properties of Conjugated Silver Nanoparticles. BP contributed Parameter Dependent Silver Nanoparticle Antibacterial Properties section of the article. JMS has guided in preparing this article, validated the content of the article, and formatted the manuscript in accordance to the guidelines for article submission. All authors have read and have approved the final manuscript for publication in International Journal of Nano Letters.

Open Access This article is distributed under the terms of the Creative Commons Attribution 4.0 International License (http://crea tivecommons.org/licenses/by/4.0/), which permits unrestricted use, distribution, and reproduction in any medium, provided you give appropriate credit to the original author(s) and the source, provide a link to the Creative Commons license, and indicate if changes were made.

\section{References}

1. Yapijakis, C.: Hippocrates of Kos, the father of clinical medicine, and Asclepiades of Bithynia, the father of molecular medicine. Rev. In Vivo (Athens, Greece) 23(4), 507-514 (2009)

2. Alexander, J.W.: History of the medical use of silver. Surg. Infect. 10(3), 289-292 (2009). doi:10.1089/sur.2008.9941

3. Yu, L., Zhang, Y., Zhang, B., Liu, J.: Enhanced antibacterial activity of silver nanoparticles/halloysite nanotubes/graphene nanocomposites with sandwich-like structure. Sci. Rep. 4 (2014). doi:10.1038/srep04551

4. Klueh, U., Wagner, V., Kelly, S., Johnson, A., Bryers, J.D.: Efficacy of silver-coated fabric to prevent bacterial colonization and subsequent device-based biofilm formation. J. Biomed. Mater. Res. 53(6), 621-631 (2000). doi:10.1002/10974636(2000)53:6<621:AID-JBM2>3.0.CO;2-Q

5. Davies, R.L., Etris, S.F.: The development and functions of silver in water purification and disease control. Catal. Today 36(1), 107-114 (1997). doi:10.1016/S0920-5861(96)00203-9

6. Yamanaka, M., Hara, K., Kudo, J.: Bactericidal actions of a silver ion solution on Escherichia coli, studied by energy-filtering transmission electron microscopy and proteomic analysis. Appl. Environ. Microbiol. 71(11), 7589-7593 (2005). doi:10.1128/aem. 71.11.7589-7593.2005

7. Slonczewski, J., Foster, J.: An Evolving Science. W.W. Norton, New York (2009)

8. Bavoil, P., Hofnung, M., Nikaido, H.: Identification of a cytoplasmic membrane-associated component of the maltose transport system of Escherichia coli. J. Biol. Chem. 255(18), 8366-8369 (1980)

9. Ong, S.Y., Wu, J., Moochhala, S.M., Tan, M.H., Lu, J.: Development of a chitosan-based wound dressing with improved hemostatic and antimicrobial properties. Biomaterials 29(32), 4323-4332 (2008). doi:10.1016/j.biomaterials.2008.07.034

10. Kollef, M.H., Afessa, B., Anzueto, A., et al.: Silver-coated endotracheal tubes and incidence of ventilator-associated 
pneumonia: the nascent randomized trial. JAMA 300(7), 805-813 (2008). doi:10.1001/jama.300.7.805

11. Li, Y., Leung, P., Yao, L., Song, Q.W., Newton, E.: Antimicrobial effect of surgical masks coated with nanoparticles. J. Hosp. Infect. 62(1), 58-63 (2006). doi:10.1016/j.jhin.2005.04.015

12. El-Rafie, M.H., Mohamed, A.A., Shaheen, T.I., Hebeish, A.: Antimicrobial effect of silver nanoparticles produced by fungal process on cotton fabrics. Carbohydr. Polym. 80(3), 779-782 (2010). doi:10.1016/j.carbpol.2009.12.028

13. Silvestry-Rodriguez, N., Sicairos-Ruelas, E., Gerba, C., Bright, K.: Silver as a disinfectant. In: Reviews of Environmental Contamination and Toxicology, vol. 191, pp. 23-45. Springer New York (2007)

14. Appendini, P., Hotchkiss, J.H.: Review of antimicrobial food packaging. Innov. Food Sci. Emerg. Technol. 3(2), 113-126 (2002). doi:10.1016/S1466-8564(02)00012-7

15. Guggenbichler, J.P., Böswald, M., Lugauer, S., Krall, T.: A new technology of microdispersed silver in polyurethane induces antimicrobial activity in central venous catheters. Infection 27(1), S16-S23 (1999). doi:10.1007/BF02561612

16. Carr, H.S., Wlodkowski, T.J., Rosenkranz, H.S.: Silver sulfadiazine: in vitro antibacterial activity. Antimicrob. Agents Chemother. 4(5), 585-587 (1973). doi:10.1128/aac.4.5.585

17. Pal, S., Yoon, E.J., Tak, Y.K., Choi, E.C., Song, J.M.: Synthesis of highly antibacterial nanocrystalline trivalent silver polydiguanide. J. Am. Chem. Soc. 131(44), 16147-16155 (2009). doi:10.1021/ja9051125

18. Moore, M.N.: Do nanoparticles present ecotoxicological risks for the health of the aquatic environment? Environ. Int. 32(8), 967-976 (2006). doi:10.1016/j.envint.2006.06.014

19. Ji, J.H., Jung, J.H., Kim, S.S., Yoon, J.-U., Park, J.D., Choi, B.S., Chung, Y.H., Kwon, I.H., Jeong, J., Han, B.S., Shin, J.H., Sung, J.H., Song, K.S., Yu, I.J.: Twenty-eight-day inhalation toxicity study of silver nanoparticles in Sprague-Dawley rats. Inhal. $\begin{array}{llll}\text { Toxicol. 19(10), } & 857-871 & \text { (2007). doi:10.1080/ }\end{array}$ 08958370701432108

20. Tsuji, T., Thang, D.H., Okazaki, Y., Nakanishi, M., Tsuboi, Y., Tsuji, M.: Preparation of silver nanoparticles by laser ablation in polyvinylpyrrolidone solutions. Appl. Surf. Sci. 254(16), 5224-5230 (2008). doi:10.1016/j.apsusc.2008.02.048

21. Wang, H., Qiao, X., Chen, J., Ding, S.: Preparation of silver nanoparticles by chemical reduction method. Colloids Surf. A 256(2-3), 111-115 (2005). doi:10.1016/j.colsurfa.2004.12.058

22. Solanki, J.N., Murthy, Z.V.P.: Controlled size silver nanoparticles synthesis with water-in-oil microemulsion method: a topical review. Ind. Eng. Chem. Res. 50(22), 12311-12323 (2011). doi:10.1021/ie201649x

23. Saion, E., Gharibshahi, E., Naghavi, K.: Size-controlled and optical properties of monodispersed silver nanoparticles synthesized by the radiolytic reduction method. Int. J. Mol. Sci. 14(4), 7880-7896 (2013)

24. Hewakuruppu, Y.L., Dombrovsky, L.A., Chen, C., Timchenko, V., Jiang, X., Baek, S., Taylor, R.A.: Plasmonic "pump-probe" method to study semi-transparent nanofluids. Appl. Opt. 52(24), 6041-6050 (2013). doi:10.1364/AO.52.006041

25. Amirjani, A., Marashi, P., Fatmehsari, D.H.: Effect of $\mathrm{AgNO}_{3}$ addition rate on aspect ratio of $\mathrm{CuCl}_{2}$-mediated synthesized silver nanowires using response surface methodology. Colloids Surf. A 444, 33-39 (2014). doi:10.1016/j.colsurfa.2013.12.033

26. Amirjani, A., Fatmehsari, D.H., Marashi, P.: Interactive effect of agitation rate and oxidative etching on growth mechanisms of silver nanowires during polyol process. J. Exp. Nanosci. 1-14 (2015). doi:10.1080/17458080.2015.1014872

27. Gurunathan, S., Kalishwaralal, K., Vaidyanathan, R., Venkataraman, D., Pandian, S.R.K., Muniyandi, J., Hariharan, N., Eom, S.H.: Biosynthesis, purification and characterization of silver nanoparticles using Escherichia coli. Colloids Surf. B 74(1), 328-335 (2009). doi:10.1016/j.colsurfb.2009.07.048

28. Liu, W., Zhou, Q., Liu, J., Fu, J., Liu, S., Jiang, G.: Environmental and biological influences on the stability of silver nanoparticles. Chin. Sci. Bull. 56(19), 2009-2015 (2011). doi:10. 1007/s11434-010-4332-8

29. Pokhrel, L.R., Dubey, B., Scheuerman, P.R.: Impacts of select organic ligands on the colloidal stability, dissolution dynamics, and toxicity of silver nanoparticles. Environ. Sci. Technol. 47(22), 12877-12885 (2013). doi:10.1021/es403462j

30. El Badawy, A.M., Scheckel, K.G., Suidan, M., Tolaymat, T.: The impact of stabilization mechanism on the aggregation kinetics of silver nanoparticles. Sci. Total Environ. 429, 325-331 (2012). doi:10.1016/j.scitotenv.2012.03.041

31. Carlson, C., Hussain, S.M., Schrand, A.M., Braydich-Stolle, L.K., Hess, K.L., Jones, R.L., Schlager, J.J.: Unique cellular interaction of silver nanoparticles: size-dependent generation of reactive oxygen species. J. Phys. Chem. B 112(43), 13608-13619 (2008). doi:10.1021/jp712087m

32. Morones, J.R., Elechiguerra, J.L., Camacho, A., Holt, K., Kouri, J.B., Ramírez, J.T., Yacaman, M.J.: The bactericidal effect of silver nanoparticles. Nanotechnology 16(10), 2346-2353 (2005). doi:10.1088/0957-4484/16/10/059

33. Panacek, A., Kvitek, L., Prucek, R., Kolar, M., Vecerova, R., Pizurova, N., Sharma, V.K., Nevecna, T., Zboril, R.: Silver colloid nanoparticles: synthesis, characterization, and their antibacterial activity. J. Phys. Chem. B 110(33), 16248-16253 (2006). doi:10.1021/jp063826h

34. Martínez-Castañón, G.A., Niño-Martínez, N., Martínez-Gutierrez, F., Martínez-Mendoza, J.R., Ruiz, F.: Synthesis and antibacterial activity of silver nanoparticles with different sizes. J. Nanopart. Res. 10(8), 1343-1348 (2008). doi:10.1007/s11051008-9428-6

35. Espinosa-Cristóbal, L.F., Martínez-Castañón, G.A., MartínezMartínez, R.E., Loyola-Rodríguez, J.P., Patiño-Marín, N., ReyesMacías, J.F., Ruiz, F.: Antibacterial effect of silver nanoparticles against Streptococcus mutans. Mater. Lett. 63(29), 2603-2606 (2009). doi:10.1016/j.matlet.2009.09.018

36. Lu, Z., Rong, K., Li, J., Yang, H., Chen, R.: Size-dependent antibacterial activities of silver nanoparticles against oral anaerobic pathogenic bacteria. J. Mater. Sci. Mater. Med. 24(6), 1465-1471 (2013). doi:10.1007/s10856-013-4894-5

37. Agnihotri, S., Mukherji, S., Mukherji, S.: Size-controlled silver nanoparticles synthesized over the range 5-100 $\mathrm{nm}$ using the same protocol and their antibacterial efficacy. RSC Adv. 4(8), 3974-3983 (2014). doi:10.1039/C3RA44507K

38. Pal, S., Tak, Y.K., Song, J.M.: Does the antibacterial activity of silver nanoparticles depend on the shape of the nanoparticle? A study of the gram-negative bacterium Escherichia coli. Appl. Environ. Microbiol. 73(6), 1712-1720 (2007). doi:10.1128/aem.02218-06

39. Berdnikova, D.V., Ihmels, H., Schonherr, H., Steuber, M., Wesner, D.: Photoinduced formation of stable Ag-nanoparticles from a ternary ligand-DNA- $\mathrm{Ag}^{+}$complex. Org. Biomol. Chem. 13(12), 3766-3770 (2015). doi:10.1039/C5OB00295H

40. Gao, J., Fu, J., Lin, C., Lin, J., Han, Y., Yu, X., Pan, C.: Formation and photoluminescence of silver nanoparticles stabilized by a two-armed polymer with a crown ether core. Langmuir 20(22), 9775-9779 (2004). doi:10.1021/la049197p

41. Vilela, D., González, M.C., Escarpa, A.: Sensing colorimetric approaches based on gold and silver nanoparticles aggregation: chemical creativity behind the assay. A review. Anal. Chim. Acta 751, 24-43 (2012). doi:10.1016/j.aca.2012.08.043

42. Ratnarathorn, N., Chailapakul, O., Henry, C.S., Dungchai, W.: Simple silver nanoparticle colorimetric sensing for copper by paper-based devices. Talanta 99, 552-557 (2012). doi:10.1016/j. talanta.2012.06.033 
43. Zhang, L., Yao, Y., Shan, J., Li, H.: Lead (II) ion detection in surface water with pM sensitivity using aza-crown-ether-modified silver nanoparticles via dynamic light scattering. Nanotechnology 22(27), 275504 (2011). doi:10.1088/0957-4484/22/27/ 275504

44. Shrivastava, S., Bera, T., Roy, A., Singh, G., Ramachandrarao, P., Dash, D.: Characterization of enhanced antibacterial effects of novel silver nanoparticles. Nanotechnology 18(22), 225103 (2007)

45. Mijakovic, I., Petranovic, D., Bottini, N., Deutscher, J., Ruhdal Jensen, P.: Protein-tyrosine phosphorylation in Bacillus subtilis. J. Mol. Microbiol. Biotechnol. 9(3-4), 189-197 (2005). doi:10. $1159 / 000089647$

46. Kora, A.J., Rastogi, L.: Enhancement of antibacterial activity of capped silver nanoparticles in combination with antibiotics, on model gram-negative and gram-positive bacteria. Bioinorg. Chem. Appl. 2013, 7 (2013). doi:10.1155/2013/871097

47. Bryaskova, R., Pencheva, D., Kyulavska, M., Bozukova, D., Debuigne, A., Detrembleur, C.: Antibacterial activity of poly(vinyl alcohol)- $b$-poly(acrylonitrile) based micelles loaded with silver nanoparticles. J. Colloid Interface Sci. 344(2), 424-428 (2010). doi:10.1016/j.jcis.2009.12.040

48. Pal, S., Yoon, E.J., Park, S.H., Choi, E.C., Song, J.M.: Metallopharmaceuticals based on silver(I) and silver(II) polydiguanide complexes: activity against burn wound pathogens. J. Antimicrob. Chemother. 65(10), 2134-2140 (2010). doi:10.1093/jac/ dkq294

49. Clark, R.F.: Overview and general considerations of wound repair. In: Clark, R.A.F., Henson, P.M. (eds.) The molecular and cellular biology of wound repair, pp. 3-33. Springer, New York (1988)

50. Tokumaru, S., Sayama, K., Shirakata, Y., Komatsuzawa, H., Ouhara, K., Hanakawa, Y., Yahata, Y., Dai, X., Tohyama, M., Nagai, H., Yang, L., Higashiyama, S., Yoshimura, A., Sugai, M., Hashimoto, K.: Induction of keratinocyte migration via transactivation of the epidermal growth factor receptor by the antimicrobial peptide LL-37. J. Immunol. (Baltimore, MD, 1950) 175(7), 4662-4668 (2005)

51. Zhou, N., Luo, Z., Luo, J., Fan, X., Cayabyab, M., Hiraoka, M., Liu, D., Han, X., Pesavento, J., Dong, C.Z., Wang, Y., An, J., Kaji, H., Sodroski, J.G., Huang, Z.: Exploring the stereochemistry of CXCR4-peptide recognition and inhibiting HIV-1 entry with D-peptides derived from chemokines. J. Biol. Chem. 277(20), 17476-17485 (2002). doi:10.1074/jbc.M202063200

52. Brennan, S.S., Foster, M.E., Leaper, D.J.: Antiseptic toxicity in wounds healing by secondary intention. J. Hosp. Infect. 8(3), 263-267 (1986)

53. Heo, D.N., Yang, D.H., Lee, J.B., Bae, M.S., Kim, J.H., Moon, S.H., Chun, H.J., Kim, C.H., Lim, H.N., Kwon, I.K.: Burn-wound healing effect of gelatin/polyurethane nanofiber scaffold containing silver-sulfadiazine. J. Biomed. Nanotechnol. 9(3), 511-515 (2013)

54. Fan, Z., Liu, B., Wang, J., Zhang, S., Lin, Q., Gong, P., Ma, L., Yang, S.: A novel wound dressing based on Ag/graphene polymer hydrogel: effectively kill bacteria and accelerate wound healing. Adv. Funct. Mater. 24(25), 3933-3943 (2014). doi:10. 1002/adfm.201304202

55. Pal, S., Tak, Y.K., Han, E., Rangasamy, S., Song, J.M.: A multifunctional composite of an antibacterial higher-valent silver metallopharmaceutical and a potent wound healing polypeptide: a combined killing and healing approach to wound care. New J. Chem. 38(8), 3889-3898 (2014). doi:10.1039/C4NJ00160E

56. Oudhoff, M.J., Bolscher, J.G., Nazmi, K., Kalay, H., van't Hof, W., Amerongen, A.V., Veerman, E.C.: Histatins are the major wound-closure stimulating factors in human saliva as identified in a cell culture assay. FASEB $\mathrm{J}$ (official publication of the Federation of American Societies for Experimental Biology) 22(11), 3805-3812 (2008). doi:10.1096/fj.08-112003 